\title{
Comprobación de los cuatro componentes de la creatividad como intangible de la mediación en México
}

\author{
Verification of the four components of creativity as intangible of \\ mediation in Mexico
}

\section{Recibido: 18-04-2021 | Aceptado: 08-07-2021}

Eni Valdes Espinosa*

*https://orcid.org/0000-0003-1483-5386

Universidad Autónoma de Nuevo León, México

\section{Resumen}

La mediación tiene un sin número de beneficios, son los intangibles en la mediación los que la componen y sostienen teórica y científicamente, dentro de estos intangibles contamos con el de la creatividad, ésta tiene presencia en las ciencias, así como en la ciencia de la mediación, por lo cual comprobaremos científicamente, para detectar cuales son los componentes que la integran, a través de una investigación mixta cualitativa y cuantitativa.

Palabras clave: Creatividad, Mediación, Intangible.

\section{Abstract}

Mediation has a number of benefits, it is the intangibles in mediation that make it up and sustain it theoretically and scientifically, within these intangibles we have creativity, this has a presence in science, as well as in the science of creativity. mediation, for which we will verify scientifically, to detect which are the components that comprise it, through a mixed qualitative and quantitative investigation.

Palabras clave: Creativity, Mediation, Intangible. 


\section{INTRODUCCIÓN}

El presente artículo inicia con los antecedentes de la mediación en México, como método de solución de conflictos y herramientas de paz, posterior a ello se abordan las fases, los modelos principales de la mediación y el perfil del mediador, así como también, se trabajan los intangibles en mediación, posterior a estos, particularmente el intangible de la creatividad, para concluir dentro de nuestro marco referencial con la mediación como herramienta de paz y la relación de la creatividad con ésta. En el apartado metodológico fueron analizados a través del método cualitativo y cuantitativo, los componentes de la creatividad como habilidad en el mediador que se describe más adelante.

La mediación, aparece en México como un mecanismo para dar solución a una problemática por la que atravesaban los tribunales al tener estos una carga excesiva de trabajo, lo que generó una saturación de casos a los cuales no se les podía dar respuesta pronta y expedita en su totalidad, del mismo modo no se contaban con los recursos suficientes para que los tribunales pudieran crecer. Estas circunstancias abrieron el camino a la mediación como mecanismo heterocompositivo, alternativo de la solución de controversias (Azar Mansur, 2003).

En el año de 1977 la mediación tomó fuerza en México, particularmente en Quintana Roo al publicarse el 14 de agosto del mismo año la nueva Ley de Justica Alternativa en dicho estado, se inauguró el Centro de Asistencia Jurídica, perteneciente al poder judicial local en donde se resolvían los conflictos sus- citados entre ciudadanos mediante procedimientos alternos a los juicios, en particular la mediación (Hernández Aguirre \& Mendivil Torres, 2015). En el estado de Baja California Sur se abrió un centro dependiente del poder judicial local en enero del 2001 donde se ofrecían los servicios de los Métodos Alternativos de Solución de Conflictos (MASC). Entre los años 2001 y 2008 se expandió el uso de la justicia alternativa entre ellos la mediación en varias entidades federativas de la república mexicana, entre ellas Nuevo León.

Fue en el 2008 cuando todos los estados del país se vieron obligados constitucionalmente a brindar mecanismos de justicia alternativa, como la mediación. El Diario Oficial de la Federación publicó el 18 de junio la reforma hecha al artículo 17 de la Constitución Políitica de los Estados Unidos Mexicanos que estableció: "las leyes preverán mecanismos alternativos de solución de controversias. En la materia penal regularán su aplicación, asegurarán la reparación del daño y establecerán los casos en los que se requerirá supervisión judicial" (Constitución Política de los Estados Unidos Mexicanos, 1917). Posterior a ello, cada estado se vio en la necesidad de desarrollar la mediación a su manera con leyes locales ajustadas a sus particularidades, facilitando con ello que la mediación pudiera implementarse en los más distintos ámbitos como el escolar, comunitario e indígena.

Así, los abogados iniciaron con la práctica de mediación en México, sabemos que el juez, el magistrado, el ministro de la corte, el litigante, el asesor jurídico y el jurista son los 
responsables de la administración de justicia. En cuanto a la procuración de justicia encontramos a los fiscales quienes también son abogados, sin embargo, encontramos auxiliares dentro de las fiscalías cuyas profesiones versan entre trabajadores sociales, criminólogos o psicólogos. Podemos también observar dentro de la administración de las políticas públicas de bienestar la presencia de todas las profesiones (Gorjón Gómez F. J., 2020).

En los últimos años, los facilitadores e investigadores en México han trabajado para que la mediación vaya incursionando en todas las ciencias difundiendo sus beneficios a otras profesiones que también trabajan en pro de la solución del conflicto, igualmente existen estudios que se refieren a los beneficios de la mediación y su tipología (Hernández \& Cabello, 2016) suscitando un ambiente multidisciplinar e interdisciplinar, por ello la ley prevé que los mediadores o administradores de los MASC pueden ser de cualquier profesión (Novel Martí, 2010), para ello será necesario que demuestren habilidades y conocimientos sobre el procedimiento de realización de los MASC. Sin embargo, la presencia del abogado se observa dentro de las políticas públicas de bienestar y las otras profesiones están presentes únicamente en el ámbito de la administración y procuración de justicia (Gorjón Gómez F. J., 2020). Hoy en día, la mediación en México ha logrado salir de la sede judicial adentrándose en variados ámbitos de la vida nacional, puesto que la participación ciudadana ha ganado fuerza mediante diversos programas (Márquez Algara \& De Villa Cortés, 2016). De esta manera, se ha logrado incursionar la mediación a los planes de estudio universitarios, a las políticas públicas a través del gobierno, brindando servicios a nivel municipal y estatal, inclusive en el sector privado (Gorjón Gómez F. J., 2017)

\section{Etapas, modelos de mediación y el me- diador}

El proceso de mediación se lleva a cabo mediante tres etapas que van perfiladas a la solución del conflicto (Gorjón \& Sanchez, Las 101 preguntas de la mediacion, 2015). De manera general, las fases se desarrollan de la siguiente manera: primero se desarrolla la de conocimiento conocida como la sesión informativa, los mediados son informados de los aspectos generales de la mediación, las partes se identifican entre sí, se identifican los temas a tratar, se elabora una agenda de trabajo y se aclaran dudas. Posterior a ello se desarrolla la etapa de comunicación, en donde se propicia una comunicación activa para que las partes generen opciones, alternativas e ideas. Por último, se trabaja la fase de formalización de acuerdo, en donde el mediador documenta el acuerdo para que lo firmen las partes. El mediador se guía por modelos de intervención básicos, cada modelo cuenta con sus objetivos particulares, su estructura y estrategias para orientar la actuación del mediador. Se mencionarán los tres modelos principales.

El Modelo Tradicional de Harvard creado por Roger Fisher, William Ury y Bruce Patton, la mediación es trabajada a manera de negociación, es asistida por un tercero. El conflicto se entiende como un impedimento para la solución de intereses o necesidades. Se enfoca en el interés de cada una de las 
partes, el conflicto surge cuando los intereses o las necesidades de los mediados son incompatibles. Se requiere diferenciar a las personas del problema.

Para poder satisfacer a ambas partes, se trabaja el tratamiento del conflicto. Se busca que las partes puedan crear opciones para beneficio mutuo. Este modelo está perfilado a alcanzar la satisfacción de los intereses de las partes a través del establecimiento de un acuerdo. Los mediadores son los expertos en derecho quienes conocen el sistema judicial, son quienes guían la interacción y discusión entre las partes buscando alcanzar un acuerdo (Quiroz Colossio, 2015).

El Modelo Transformativo ideado por Robert A. Baruch Bush y Joseph P. Folger, se diferencia del modelo Harvard al denominarse historia de la transformación a diferencia del modelo anterior al cual denominan historia de la satisfacción. El objetivo de la mediación está perfilado a desarrollar el potencial de cambio de los mediados al descubrir sus habilidades personales en el transcurso del procedimiento de mediación. Suscita el desarrollo moral centrándose en la mejora o transformación de las relaciones humanas. Este modelo se centra en trabajar el reconocimiento y la revalorización de cada una de las partes. (Gimenéz, 2016).

El Modelo Circular-Narrativo fue elaborado por Sara Cobb quien enfoca su trabajo en narraciones de las personas durante el procedimiento de mediación. Su objetivo es lograr un acuerdo a través de la interacción y comunicación entre las partes. Para esto se requiere desarrollar la historia conflictiva con la que llegan las partes buscando una mediación, con la finalidad de historizar, analizando la historia, tratando de comprender cómo están entendiendo los hechos, las actitudes de cada uno y las relaciones entre ellos.

Se requiere cambiar la narrativa con la que las partes acuden a la mediación, para poder reacomodar la relación confrontativa, facilitando que las partes puedan generar una historia diferente que posibilite el cambio. Para ello el mediador requiere introducir caos, posterior a esto acomoda las posiciones generando la oportunidad de que las partes puedan hablar de forma distinta a como lo han venido haciendo, con la finalidad de que se generen cambios, los cuales crean la posibilidad de arribar a un acuerdo (Gorjón \& Sanchez, Las 101 preguntas de la mediacion, 2015).

Encontramos una amplia variedad de acepciones en relación con el mediador, demudan respecto al tipo y modelo de mediación, de acuerdo con el autor, el país, la escuela que lo define, la normativa o bien el entorno del conflicto ya sea público o privado. Gorjón y Sánchez (2016), presentan un concepto simple y concreto, consideran que "el mediador es un tercero que facilita a las partes en conflicto a ponerse de acuerdo y solucionar sus conflictos", de igual modo, enuncian los cuatro fundamentos legales que lo sostienen:

- Artículo 2.XII, 18, 20, 40, 41 y 47 de la Ley de Justicia Alternativa del Tribunal Superior de Justicia para el Distrito Federal (TMX 258019). 
- Artículo 2.IX de la Ley de los Métodos Alternos de Solución de Conflictos del Estado de Nuevo León (TMX 295622).

- Artículo 3.V de la Ley Nacional de Mecanismos Alternativos de Solución de Controversias en Materia Penal (TMX 844369).

- Artículo 60 y 65 del Reglamento Interno del Centro de Justicia Alternativa del Tribunal Superior de Justicia de la Ciudad de México

Steele (2015), sostiene que un mediador requiere ser transparente y objetivo en la mediación, conservando una posición equilibrada ante la postura de ambas partes, tiene como propósito aclarar los aspectos imprecisos del conflicto a efecto de que este sea entendido en su justa dimensión, puntualizando en que ambas partes están de acuerdo para lograr una solución al mismo, basándose en los datos proporcionados por los mediados.

Así mismo, Márquez (2013), plantea que el mediador es un profesional especializado en la resolución de conflictos, al recibir una formación en el ámbito y el dominio de técnicas específicas de resolución de conflictos. Como lo afirma Pastor e Iglesias (2011), la formación sólida de un mediador sustentada en técnicas, habilidades, procesos de mediación, además de lo teórico - práctico es lo que transformara al mediador en un competente profesional, porque dispone de lo necesario para ser eficaz y eficiente en su trabajo.

Por lo tanto, el mediador es el profesional capacitado específicamente en esta área.
Lleva a cabo una función de facilitador, por lo cual, él no es quien decide o impone la solución, más bien es quien acompaña a las partes a comunicarse para gestionar su conflicto. Dentro del procedimiento de mediación, el mediador promueve el diálogo abierto entre las partes, escucha atentamente la discusión centrada en los intereses, las preocupaciones y los problemas de los mediados con la finalidad de buscar soluciones creativas para llegar a un acuerdo mutuo. De esta manera, el mediador ejecuta sus funciones profesionales con fundamento en su experiencia, conocimientos, entrenamiento, práctica, inteligencia, técnicas, habilidades y cualidades que ha desarrollado. Igualmente, el mediador pone en práctica estrategias y técnicas que le permitan la modificación de actitudes entre las partes. Mediante una participación de oyente activo, el mediador genera ideas entre las partes con la finalidad de arribar a un acuerdo. El trabajo del mediador está enfocado en facilitar que las partes logren un acuerdo en donde los términos sean satisfactorios para cada una de ellas.

Dupuis citado en (Gorjón \& Sáenz, 2009), enuncian dos características básicas que debe tener el mediador: 1. La imparcialidad, alude a que el mediador no debe externar opiniones tendenciosas o inclinarse por alguna de los mediados, en otras palabras, no debe tomar partido a favor de ninguna de las partes. 2. La neutralidad, apunta al comportamiento y a la relación entre el mediador y las partes. El mediador requiere diferenciar sus opiniones de las de los mediados, así como también, acompañarlos a formular sus ideas y tomar sus decisiones. Es prescindible 
que esté atento a no dejarse contaminar en el conflicto. Siguiendo con esta línea (Steele, 2015) suma a lo anterior diez características que debe reunir el perfil de un mediador: 1. Dar confianza y seguridad a los mediados, 2. Fungir como puente de comunicación, 3. Ser buen observador, 4. En caso de crisis poder reconducir las situaciones a términos objetivos, 5. Capacidad para sintetizar los argumentos brindados por las partes, 6 . Convicción para que las partes arriben al acuerdo, 7. Tener imaginación, creatividad y poder improvisar, 8. Escuchar activamente, 9. Ser paciente y tolerante, y 10. Contar con sentido del humor.

\section{Los intangibles en mediación}

Estos presentan un interés focal por difundir el conocimiento técnico-científico, y traducirlo a un conocimiento más coloquial, se vuelve una estrategia de culturización de la implementación de los MASC buscando redimensionarlos y poder transmitir sus beneficios a la sociedad a través de su valor real. Para Sáenz (2015), que explica que no existía una cultura de implementación en la solución de conflictos ya que persiste un desconocimiento de su valor, desconocimiento de las estrategias de comunicación, de promoción y de educación, que se centraban en conceptos más técnicos dejando a un lado sus intangibles.

Gorjón (2013), plantea la necesidad de que la comunidad científica de los MASC desarrolle cambios profundos, redimensionando sus principios, características, tomando en cuenta el valor intangible de los mismos, esto como estrategia de culturización subra- yando el valor positivo de sus resultados, así como el impacto que tiene en la comunidad, donde los beneficios sean fácilmente reconocidos.

Los intangibles en mediación están fundamentados en la literatura empresarial y respaldados en teorías administrativas, cuenta con elementos sustantivos, de composición como el ejercicio intelectual, el capital estructural, el capital humano y el capital relacional los cuales se interrelacionan a lo largo del procedimiento de gestión de cualquier conflicto, buscando lograr su trasformación (Gorjón Gómez F. J., 2017). Plantea que la mediación se sustenta en su capital intelectual, además de tratarse de un valor económico también y sobre todo se trata de un ejercicio de gestión del conocimiento, de un ejercicio intelectual, que es innovador, así como creativo trascendiendo las fronteras de lo tangible basándose en los intereses de los involucrados en el conflicto para construir una solución apropiada a cada caso particular.

Sabemos que los elementos instrumentales de la mediación son las habilidades, los conocimientos, así como los valores, los esquemas operativos diferenciados, también las aptitudes de los mediadores y, por último, pero no menos importante, la relación con los usuarios, todos estos elementos se sustentan en el capital intelectual, al igual que los intangibles, que a su vez se integran por las áreas del capital humano, el capital estructural y el capital relacional. Para Gorjón (2017), que vincula directamente a la mediación con el capital intelectual cuando explica que el capital estructural brinda la 
estabilidad de su implementación ubicado en los reglamentos, normas y administradores, el capital humano son los componentes profesionales y específicos de mediadores en pro de la solución del conflicto, el capital relacional genera el vínculo de la mediación con la comunidad, con los usuarios, otorgando beneficios y condiciones de culturización mediante su percepción.

Los intangibles tendrán una connotación positiva y cuentan con un valor intrínseco en la mediación, son 43 intangibles de la mediación propuestos por Gorjón (2017), a continuación, se enlista cada uno de ellos:

1. Activo de paz

2. Armonía

3. Asociatividad

4. Autonomía

5. Bajo costo

6. Capital social

7. Cláusula de estilo

8. Competitividad

9. Confianza

10. Confidencialidad

11. Creatividad

12. Empoderamiento

13. Especialización

14. Estabilidad

15. Felicidad

16. Fidelización

17. Flexibilidad

18. Garantía

19. Independencia

20. Innovación

21. Internacionalización

22. Liderazgo

23. Mejora continua

24. Modernidad
25. Monopolización del procedimiento por las partes

26. Motivación

27. Neutralidad

28. No trae consecuencias económico-sociales

29. Objetividad

30.Perdón

31. Prestigio

32. Prevención del conflicto

33. Productividad

34. Rapidez

35. Reasociación

36. Reparación del daño

37. Reputación corporativa

38. Satisfacción

39. Seguridad jurídica

40. Simplicidad, evita la complejidad

41. Sinergia

42. Transparencia

43. Universalidad

Con esto tendríamos un punto de partida al intangible de la creatividad porque lo delimita y le da un lugar, también es importante señalar que todos los intangibles están relacionados unos con otros, si bien fue de nuestro interés investigar el intangible de la creatividad, no podemos negar la relación estrecha con los otros 42.

Desde la escuela de pensamiento, precursores de los métodos alternos de solución de conflictos siguen trabajando investigaciones, proyectos de tesis para comprobar cada uno de los 43 intangibles que existen, se han comprobado hasta la presente investigación el intangible del perdón, el de la felicidad, el del bienestar y el de la confianza. Intangible de la creatividad en mediación 
La creatividad como intangible de la medición produce un efecto positivo, puesto que es capaz de generar valor relacionado con:

\section{Tabla 1. Intangible de la creatividad, efecto positivo.}

\begin{tabular}{|l|l|}
\hline $\begin{array}{l}\text { SUJETO DEL EFECTO } \\
\text { POSITIVO }\end{array}$ & EFECTO POSITIVO \\
\hline $\begin{array}{l}\text { Mediados } \\
\text { Mediador }\end{array}$ & $\begin{array}{l}\text { 1. El proceso de mediación, en el cual, el mediador crea un ambiente } \\
\text { de confianza con los mediados y de los mediados con el proceso, así } \\
\text { como también la relación de confianza, respeto, escucha, disposi- } \\
\text { ción, apertura, entre los mediados. }\end{array}$ \\
\hline Mediados & $\begin{array}{l}\text { 2. Los mediados, es decir, la manera en cómo resuelven sus proble- } \\
\text { mas creativamente y obtienen un beneficio directo. }\end{array}$ \\
\hline Mediador & $\begin{array}{l}\text { 3. El mediador, al utilizar la creatividad, como habilidad para llevar a } \\
\text { cabo diversas actividades, encaminadas a la solución del conflicto, } \\
\text { para arribar al acuerdo de mediación. }\end{array}$ \\
\hline
\end{tabular}

Fuente: Elaboración propia.

Representa un tema poco explorado científicamente en el campo de la mediación el intangible de la creatividad, siendo de nuestro interés expresar los beneficios que se obtienen con los procesos de mediación, así como teorizarlos, sistematizarlos y medirlos, con el motivante de contribuir a la construcción de la ciencia de la mediación.

La creatividad como intangible de la mediación atrae nuestra atención ya que ha sido poco estudiado e investigado a profundidad por otros científicos. Se ha planteado la existencia de la creatividad en la mediación como intangible, como ya se ha señalado en el presente artículo por el Dr. Gorjón en su obra Mediación, su valor intangible y efectos operativos. El autor nos dice que para encontrar soluciones en el proceso de mediación se requiere desarrollar opciones las cuales surgen de la creatividad de las personas involucradas en el problema (Gorjón, 2017). Si bien investigar la creatividad como intangible de la mediación no es propiamente una teoría por sí sola, el analizarla desde un punto de vista científico permite la teorización de los intangibles para que algún día éste se convierta 
en un paradigma. Analizar la creatividad como habilidad del mediador dentro del proceso de mediación en los MASC, permite que se forjen nuevas formas de solución de conflictos, mejorando la pacificación de una forma más inmediata, y creando alternativas para enfrentar los conflictos, y con ello despresurizar los sistemas de administración de justicia, por lo cual se tiene que seguir investigando la presencia de forma científica de la creatividad en mediación (Gorjón \& Valdés-Espinosa, 2020).

\section{La mediación como herramienta de paz y su relación con la creatividad}

A lo largo de la historia el concepto de paz se ha definido, estudiado y trabajado contribuyendo a su evolución. En el mundo occidental los griegos entendían la paz como la ausencia de la guerra o el estado de armonía. Por su parte los romanos sostenían que la paz era la ausencia de violencia. Sin embargo, en el oriente, la idea de paz discrepó por razones culturales y políticas. Las culturas y religiones orientales plantean que se llega a la paz mediante la tranquilidad y equilibrio interno de las personas y de éstas con su entorno.

Gorjón y Sánchez (2016), definen que:

la paz es un espacio de tiempo de tranquilidad, es un estadio de calma, es un estatus de serenidad, en donde las personas se desarrollan plenamente, en el cual las personas viven o trabajan en plenitud, debido a sus propios intereses y estilo de vida.
La cultura de paz se ha abordado desde diversos ámbitos, y variados personajes han sobresalido por las aportaciones realizadas, así, Rigoberta Menchú, líder indígena guatemalteca, premio Nobel de la Paz en 1992, concibe a la paz como hija de la convivencia, de la educación y del diálogo. Sostiene que la Paz debe ser la construcción de un proceso de armonía social, económico, jurídico y político en el que la sociedad se sienta incluida, respetada y reconocida (López Palomar, 2015). La mediación juega un rol significativo en esto, en tanto que se da en un ambiente de respeto, se promueve que sean las partes involucradas quienes mediante el diálogo puedan crear una solución al conflicto alcanzando como consecuencia un estado de paz.

Así mismo, el sociólogo y matemático Johan Galtung, pacifista noruego y fundador de los estudios de Paz, sostuvo que, tras la violencia directa, se persigue una reconstrucción para reparar los daños causados; tras la violencia cultural, se busca alcanzar una reconciliación de las partes en conflicto y tras la violencia estructural, se pretende lograr una resolución de las desigualdades, de las contradicciones que propician la violencia directa (Concha, 2009). Uno de los medios para alcanzar la paz es la mediación, en donde se trabaja para arribar a la resolución del conflicto, las partes pueden acordar la reparación del daño y en ocasiones se logra un efecto de reconciliación entre las partes.

Por su parte, el negociador de conflictos y escritor norteamericano John Paul Lederach, trabaja para crear nuevos caminos para la 
construcción de la paz. Precisa que para pensar estrategias en la construcción de paz es necesario pensar en lo que da la vida y en lo que mantiene vivas las cosas, es decir, que para ser estratégico se debe creer en algo más allá de lo existente a partir de lo que está disponible, puntualizando en su potencial exponencial.

En relación con el cambio social, refiere la necesidad de desarrollar una capacidad para reconocer y construir el locus del potencial para el cambio (Lederach, 2015). Trasladando esto a la mediación, damos cuenta que el mediador acompaña a las partes en la búsqueda de nuevos caminos para la solución del conflicto, guía a las partes para que descubran que existen posibilidades y que juntos pueden crear opciones para solucionarlo.

En el caso del ex presidente de Costa Rica Oscar Arias Sánchez, Premio Nobel de la Paz 1987, afirma que la paz no es el fruto del esfuerzo de una persona, de un grupo o siquiera de una generación, la paz es un bien colectivo, que se mantiene por el esfuerzo de todos, también se debilita por la indiferencia de todos (Arias Sánchez, 2012). Esto se facilita al utilizar la mediación, sabemos que esta no se limita solamente a la administración de justicia, también fomenta la participación ciudadana y, además, se puede pensar en la paz como un bien colectivo.

El director de la Escuela de Cultura de la Paz de la Universidad Autónoma de Barcelona y titular de la Cátedra Unesco sobre Paz y Derechos Humanos, Dr. Vicenç Fisas Armengol, reflexiona que la paz es algo más que la ausencia de guerra, y no se logra a través de la guerra, por el contrario, tiene que ver con la superación o reducción de todo tipo de violencia física, estructural, psicológica o ecológica (Rendón Merino, 2000).

Por su parte, el Dr. Paris A. Cabello-Tijerina mexicano profesor investigador, considera la mediación como una política social que si es implementada como tal se estaría beneficiando a una gran parte de las sociedades modernas, que demandan procedimientos eficaces para transformar los conflictos de manera positiva, devolviendo un rol activo a las partes en conflicto, y así cumplir con el paradigma jurídico actual. La paz se ha identificado como la condición óptima de todos los seres humanos, se ha convertido en un objetivo que se pretende alcanzar (Cabello, Carmona, Gorjón, Sáenz, \& Vázquez, 2016).

De las investigaciones propuestas por Cabello (2012), la mediación es una vía eficaz para la consecución y fortalecimiento de una cultura de paz, auxilia a fundar un entorno pacífico, ayuda a transformar los conflictos en ideas, fomenta el diálogo, la empatía, la solidaridad, la no-violencia, la comprensión, la integración, la creatividad, la participación y el consenso, elementos de carácter sine qua non de la Paz, por lo que la mediación se considera una actividad estratégica de la cultura de la paz. Gorjón (2017), considera que la mediación es un activo de paz, visto desde la perspectiva de su intangibilidad, ya que provoca bienestar a las personas y a la sociedad, y se posiciona como un instrumento clave para el desarrollo de la cultura de la no violencia, la prevención 
y la solución de conflictos. Mientras que la creatividad otro actor central también está presente en la resolución de conflictos como los comunitarios, los electorales (Vera \& Valdés-Espinosa, 2021), entre otros.

\section{METODOLOGÍA}

El método utilizado en esta investigación fue mixto, es decir, cualitativo y cuantitativo, el cualitativo se llevó a cabo mediante la entrevista semiestructurada que consta de 12 preguntas abiertas aplicada de manera individual, presencial y en el lugar de trabajo de cada participante. Para el método cuantitativo se elaboró una encuesta de 37 ítems con escala Likert de respuestas sistemáticas de conocimiento, aplicada de manera virtual por medio de Google forms.

\section{Población}

En el estudio cualitativo, para la realización de la entrevista semiestructurada, la muestra poblacional estuvo conformada por nueve personas: tres hombres y seis mujeres. El criterio de selección se basó en que las personas tuvieran más de 12 años de experiencia en el campo operativo y/o académico en mediación, es decir, que fueran expertos en mediación, académicos MASC y capacitadores de mediadores del sector público y privado del estado de Nuevo León. Los nueve participantes poseen un promedio general de 16.7 años de experiencia.

La población analizada bajo el estudio cuantitativo, a través de la encuesta con escala Likert, cuenta con una muestra que se compone por 61 mediadores tanto públicos, como privados, certificados ante el Po- der Judicial del Estado de Nuevo León en el 2018 , de los cuales 28 fueron hombres y 33 mujeres, 17 de los participantes pertenecían al Instituto de Mecanismos Alternativos del Estado de Nuevo León, 35 pertenecientes a la Fiscalía del Poder Judicial del Estado de Nuevo León y 19 al Colegio de Mediadores.

\section{PROCEDIMIENTO}

En el análisis cualitativo de los resultados se utilizó como procedimiento una tabla para cada una de las cuatro variables se construyó una categorización de éstas y se analizaron bajo el modelo de codificación presentado por (Sáenz, 2014). Se realizó la transcripción de las nueve entrevistas, a manera de operacionalización de cada variable, se buscó manualmente dentro de las respuestas de los entrevistados las palabras asociadas, vinculadas a ellas. Se utilizó el programa MAXQDA 2020, mediante la herramienta de codificación inteligente, para corroborar que todas las subcategorías identificadas de manera manual con el método anterior y sus códigos se encontraban, dentro de las respuestas de las nueve entrevistas.

El procedimiento cuantitativo para el estudio de los resultados utilizado fue un análisis factorial a través de un revisión de los componentes principales y un análisis estadístico descriptivo. El enfoque cuantitativo de la investigación fue de carácter no experimental puesto que se estudia a una sola muestra y cuenta con un diseño transversal, ya que se utilizó el mecanismo de la encuesta, donde se tuvo un solo momento. Siendo de corte no predictivo. Posterior a esto se efectuó un análisis de triangulación de datos. 


\section{RESULTADOS}

De acuerdo con nuestro instrumento cualitativo y en el instrumento cuantitativo logramos identificar los componentes de la creatividad como una de las más importantes habilidades del mediador, como se muestra en las siguientes gráficas.

\section{Gráfica 1. Resultados de la identificación de los componentes de la creatividad en mediación cualitativo.}

\section{Cualitativo \\ Componentes de la Creatividad}

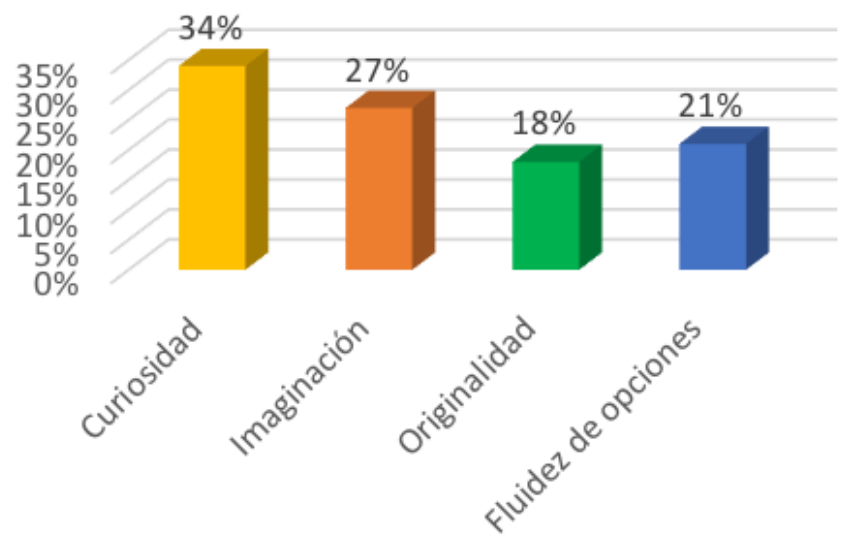

Fuente: Elaboración propia.

\section{Gráfica 2. Resultados de la identificación}

\section{Cuantitativo}

Componentes de la Creatividad

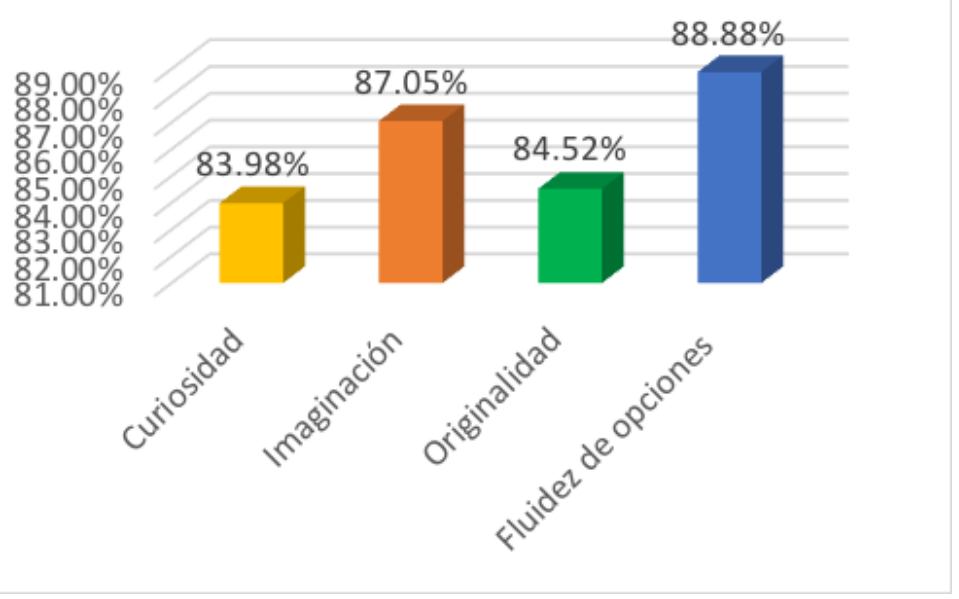

Fuente: Elaboración propia. 
Estas gráficas también responden al objeto de nuestra investigación que fue delimitar la influencia de cada uno de los componentes de la creatividad encontrando que para el instrumento cualitativo la curiosidad tiene mayor influencia en la creatividad y para el instrumento cuantitativo como ya lo mencionamos fue la fluidez de opciones. En ambos casos la imaginación y la originalidad se posicionan en el mismo orden de influencia, al describir cómo opera cada uno de los componentes de la creatividad, encontramos múltiples coincidencias, en el cualitativo utilizando la modalidad de categorización de información para el análisis de las entrevistas, que se trabajó desde una categoría, una subcategoría y palabras asociadas.

En la variable curiosidad en el enfoque cualitativo, nos encontramos con un 37\% para descubrir información y abierto a nuevas ideas con el mismo porcentaje y en tercer lugar con $26 \%$ indagar información. En el cuantitativo de igual forma, abierto a nuevas ideas alcanza los ítems con mayor porcentaje, seguido por descubrir información y de nuevo en tercer lugar indagar información con menor porcentaje.

En cuanto a la variable imaginación, encontramos pensar distinto y representación mental con $34 \%$ y en tercer lugar con $32 \%$ de posibilidades, esto en el cualitativo.

Del mismo modo en el cuantitativo, pensar distinto seguido por representación mental tienen los más altos valores y de nuevo en tercer lugar los ítems de posibilidades.
La variable originalidad desde el instrumento cualitativo nos indica con un $42 \%$ nuevas ideas seguido con un $28 \%$ ideas inusuales y en tercer lugar con un 30\% innovación. De nuevo en lo cuantitativo se corroboran estos datos, en primer lugar, nuevas ideas seguido por ideas inusuales y en tercer sitio innovación.

Fluidez de opciones nuestra última variable arrojó en primer lugar con 39\% comunicación, seguido por cantidad de ideas y en tercer sitio no criticar. Para el instrumento cuantitativo de igual forma comunicación, cantidad de ideas y no criticar fue el orden en ponderación. Estas similitudes nos permiten aseverar que respondimos a nuestro objetivo al que ahora lo analizamos de forma mixta y poder comprobarlo.

En cuanto a la investigación cuantitativa, cada ítem de cada uno de los cuatro componentes obtuvo un porcentaje mayor al $75 \%$, lo que nos hace evaluar la existencia como habilidad del mediador, nos planteamos encontrar la presencia de la creatividad y sus cuatro componentes en mediación. Se llevó a efecto el análisis de triangulación también para identificar contradicciones (Bamberger, 2020). Hubo una inversión de porcentajes que van de mayor a menor frecuencia en los cuatro componentes de la creatividad, en los resultados cualitativos y en los resultados cuantitativos.

Esta inversión de lugares en el cualitativo y el cuantitativo lo podemos explicar de la siguiente manera, considerando que la palabra fluidez de opciones tiene un tinte más académico y científico que el término curio- 
sidad, que se relaciona más con la infancia o lo popular. Tomando en cuenta que en el cuantitativo fue un trabajo de mediadores con mayor volumen de participantes, y que en el cualitativo son expertos, académicos y capacitadores en mediación donde el volumen fue menor.

\section{CONCLUSIONES}

Cuando utilizamos de forma aislada una idea de investigación dentro de los métodos cualitativos o cuantitativos tendremos ciertas ventajas o desventajas. Nuestra investigación es aprovechar las ventajas que nos da un análisis mixto, como las reflexiones que ya desarrollamos. Sabemos que los métodos cuantitativos son idóneos para la recolección de muestras y análisis de datos numéricos, pero los métodos cualitativos también son herramientas eficaces para dicho ejercicio como lo comprobamos en esta investigación. La intención para haber realizado esta triangulación de hallazgos, además de aumentar nuestra validez y credibilidad, es poder hacer un ejercicio propositivo con la información obtenida, y con ello poder comparar los datos cualitativos y cuantitativos.

Logramos también incorporar una variedad de análisis que fortalezcan la presencia y la existencia de la creatividad en la mediación sostenida por sus cuatro componentes principales. En esta investigación no hubo un enfoque predominante, tanto el cualitativo, como el cuantitativo nos permitió obtener datos duros que demuestran la validez de la teoría de los intangibles en la mediación propuesta por (Gorjón, 2017), y en nuestro caso la presencia relevante de la creatividad.
Esta triangulación nos permite dar cuenta de la viabilidad las teorías trabajadas durante este análisis están soportadas en nuestro marco referencial bibliográfico así como por los resultados arrojados por el método utilizado, tanto en lo cualitativo y como en lo cuantitativo. Nuestros hallazgos permitirán promover propuestas y conclusiones que vayan orientados a promover cursos de capacitación en la materia, y muy probablemente a introducir reformas a las leyes de métodos de solución de conflictos que mejoren su eficacia, entre otras cosas.

Finalmente cabe remarcar a los componentes de la creatividad como habilidad fundamental del mediador los que de forma intangible integran la mediación son: la curiosidad, la imaginación, la originalidad y la fluidez de opciones.

\section{TRABAJOS CITADOS}

Arias Sánchez, O. R. (13 de agosto de 2012). La utopía de una convivencia en paz. XLIX Cátedra de las Américas: a 25 Años de los Acuerdos de Esquipulas. Washington D.C.: Organización de Estados Americanos. Recuperado en: http://www.oas.org/es/sre/dai/catedra/catedras/ xlix_catedra_centroamerica/discurso\%20presidente $\% 20$ arias $\% 20$ - $\% 20$ catedra $\% 20$ de $\% 20$ las $\% 20$ americas\%20_2_.pdf

Azar Mansur, C. (2003). Mediación y conciliación en México: dos vías alternas de solución de conflictos a considerar. México, D.F.: Porrúa.

Bamberger, M. (03 de agosto de 2020). Introducción a los Métodos Mixtos de la Evaluación de Impacto. Notas sobre la Evaluación de Impacto. Guía de Orientación (3) Recuperado en: https://www.interaction.org/wp-content/uploads/2019/04/3-Mixed-Methods-in-ImpactEvaluation-SPANISH.pdf 
Brooking, A. (1997). El capital intelectual. El principal activo de las empresas del tercer milenio. Barcelona: Paidós Ibérica.

Cabello Tijerina, P. A.(2012). La mediación como política social aplicada al fortalecimiento de la cultura de paz en México y España. Tesis Doctoral. Murcia: Universidad de Murcia. Recuperado en: http://eprints.uanl.mx/4389/1/ tesis.pdf

Cabello Tijerina, P., A., Carmona Valdés, S.E., Gorjón Gómez, F.J., Sáenz López, K. A., \& Vázquez Gutiérrez, R. L. (2016). Cultura de paz. Ciudad de México: Grupo Editorial Patria.

Calderón Concha, P. C. (2009). Teoría de conflictos de Johan Galtung. Revista de Paz y conflictos (2) pp. 60-81. Recuperado en: https://revistaseug.ugr.es/index.php/ revpaz/article/view/432/477

Constitución Política de los Estados Unidos Mexicanos. (1917). Diario Oficial de la fedeación, 5(II).

De Luna Noyola, A. G. (2018). Capital Humano Gestión por competencias laborales en la administración pública. Ciudad de México: Trillas.

Dupuis, J. C. (1997). Mediación y Conciliación. Buenos Aires: Abeledo - Perrot.

Edvinsson, L., y Malone, M.S. (1999). El capital intelectual. Cómo identificar y calcular el valor de los recursos intangibles de su empresa. Barcelona: Gestión 2000.

Gimenéz Romero, C. (2001). Modelos de mediación y su aplicación en mediación intercultural. Migraciones. Revista del Instituto Universitario de Estudios sobre Migraciones, (10), 59-110. Recuperado en: https://revistas. comillas.edu/index.php/revistamigraciones/article/ view/4376/4197

Gorjón Gómez, F. J. (2013). Los intangibles de la mediación en Gorjón Gómez F.J. y López Peláez A. Estado del Arte de la Mediación (pp. 147-177). Pamplona, Navarra, España: Thomas Reuters Aranzadi.

------- (2015). Las 101 preguntas de la mediación. México, D.F.: Tirant Lo Blanch.

-- (2017). Mediación, su valor intangible y efectos operativos. Una vision integradora de los métodos alternos de solución de conflictos. Ciudad de México: Tirant Lo Blanch.

-------- (2020). La mediación como vía al bienestar y la felicidad. Ciudad de México: Tirant Lo Blanch.

Gorjón Gómez, F. J., y Sáenz López, K. A. (2009). Métodos Alternos de Solución de Controversias. Enfoque Educativo por Competencias.México, D.F.: Patria.

Gorjón Gómez, F. J. \& Pesqueira Leal, J. (2015). La ciencia de la mediación. México, D.F.: Tirant Lo Blanch.

Gorjón Gómez, F.J., \& Stelle Garza, J. G. (2015). Métodos alternativos de solución de conflictos. México, D.F.: Oxford. Gorjón Gómez, F., J. \& Sánchez García, A. (2016). Vademécum de Mediación y Arbitraje. Ciudad de México: Tirant Lo Blanch.

Gorjón Gómez, F.J., \& Valdés-Espinosa, E. (2020). Análisis de la creartividad de la mediación. Revista Ciencia Jurídica y Política, 12 (6) pp. 14-27. Recuperado en: https:// portalderevistas.upoli.edu.ni/index.php/5revciencasjuridicasypoliticas/article/view/641/67

Gorjón Gómez, G. J. (2016). Mediación: El paradigma jurídico del siglo XXI en Cabello Tijerina P. A. en La multidisciplinariedad y sus ámbitos de aplicación (pp. 71-87). México: Tirant Lo Blanch.

Hernández Aguirre, C.N., \& Mendivil Torres, J. \& y Hernández Aguirre, C. V. (2015). Importancia de los métodos alternativos de solución de controversias en el sistema penal acusatorio mexicano. Revista Ciencia Jurídica 4 (7), pp. 71-90. Recuperado en: http://www.cienciajuridica.ugto.mx/index.php/CJ/article/view/126/121

Hernández Castillo, G.D., \& y Cabello Tijerina, P. A. (2016). Tipología de los beneficios de la mediación. Azarbe Revista Internacional de Trabajo Social y Bienestar, (5) pp. 99-78. Recuperado en: https://revistas.um.es/azarbe/ article/view/266001/200271

Highton, E. y Álvarez, G.(2004). Mediación para resolver conflictos. Buenos Aires: AD-HOC.

Lederach, J. P. (2015). El arte y el alma de construir la paz. Bogotá: Grupo Editorial Norma.

Ley de Mecanismos Alternativos para la Solución de Controversias para el Estado de Nuevo León (05 de marzo 
de 2018). H. Congreso del Estado de Nuevo León. Recuperado de http://www.hcnl.gob.mx/trabajo_legislativo/ leyes/pdf/LEY\%20DE\%20MECANISMOS\%20ALTERNATIVOS\%20PARA\%20LA\%20SOLUCION\%20DE\%20 CONTROVERSIAS\%20PARA\%20EL\%20ESTADO\%20 DE\%20NUEVO\%20LEON.pdf

Ley Nacional de Mecanismos Alternativos de Solución de Controversias en Materia Penal del 2014, Capítulo 2, artículo 21. (20 de marzo de 2018). Ciudad de México. Recuperado en: http://www.diputados.gob.mx/LeyesBiblio/pdf/LNMASCMP_291214.pdf

López I Palomar, A. (2015). Rigoberta Menchú: aportaciones a la construcción de una cultura para la paz Tikb' al Utzill, que significa "sembrando paz". Fórum de recerca, (20), pp. 19-32. Recuperado en: http://repositori.uji.es/ xmlui/bitstream/handle/10234/172564/Lopez_Palomar. pdf?sequence $=1$ \&isAllowed $=y$

Márquez Algara, M. G., y De Villa Cortés, J. C. (2016). Mediación y Participación Ciudadana en México. lus $\mathrm{Hu}$ mani. Revista de Derecho, (5), pp. 45-68. Recuperado en: https://vlex.ec/vid/mediacion-participacion-ciudadana-mexico-663821729

Marques Cebola, C. (2013). La mediación. Madrid: Marcial Pons.

Novel Martí, G. (2010). Mediación organizacional: desarroIlando un modelo de éxito compartido.Madrid: Reus.

Ordóñez de Pablos, P. (2004). El Capital Estructural Organizativo como fuente de competitividad empresarial: un estudio de indicadores. Revista Economia Industrial, (357) pp. 131-140. Recuperado en: https://www.mincotur. gob.es/Publicaciones/Publicacionesperiodicas/Economialndustrial/RevistaEconomialndustrial/357/13_PatriciaOrdonez_357.pdf

Pastor Seller, E., \& Iglesias Ortuño, E. (Enero-Junio de 2011). La mediación intrajudicial como método de resolución de conflictos en el seno familiar. Entramado Revista Científica Internacional, 7(1), pp. 83-84. Recuperado en: https://revistas.unilibre.edu.co/index.php/entramado/ article/view/3391/2783
Quiroz Colossio, L. I.(2015). La construcción teórica de la ciencia de la mediación a partir de los modelos instrumentales existentes (pp. 93-118) en Gorjón Gómez F.J., \& Pesqueira Leal, J. La ciencia de la mediación. México, D.F.: Tirant Lo Blanch.

Ramos Morales, L. A. (2017). Los Elementos del Perdón en la Mediación Familiar. Tesis Doctoral Facultad de Derecho y Criminología. Recuperado en: http://eprints.uanl. $\mathrm{mx} / 13900 / 1 / 1080216035 . p d f$

Rendón Merino, Á. (2000). El aprendizaje de la paz: métodos y técnicas de su construcción desde procesos pedagógicos.Santa Fe de Bogotá: Cooperativa Editorial Magisterio.

Rodríguez Rodríguez, M. E. (2018). Principales modelos de mediación y de métodos alternos (mediación, conciliación) en Gorjón Gómez F. J., \& Chávez de los Ríos R. Manual de mediación penal, civil, familiar y justicia restaurativa, para mediadores, facilitadores e instructores (pp.73-82). Ciudad de México: Tirant Lo Blanch.

Sáenz López, K. A. (2015). La epistemología de la ciencia de la mediación en Gorjón Gómez F. J, \& Pesqueira Leal, J. La ciencia de la mediación (pp. 57-71). México, D.F.: Tirant Lo Blanch.

Scarabino, J.C. (2000). Activos intangibles. El puente hacia la contabilidad de concimiento.. Santa Fe Argentina: Consejo Profesional de Ciencias Económicas.

Scarabino, J. C., Biancardi, G., \& Blando, A. (2007). Capital Intelectual. Invenio, Revista de Investigación Académica 10 (19), pp. 59-71. Recuperado en: https://www.redalyc. org/pdf/877/87701905.pdf

Steele Garza, J. (2015). La mediación en las relaciones de consumo en Cabello Tijerina P.A., La multidisciplinariedad de la medlación y sus ámbitos de aplicación (pp.89-106). México, D.F.: Tirant Lo Blanch.

Urquidi, J. E. (1999). Mediación Solución a Conflictos sin Litigio. México, D.F.: Centro de Resolución de Conflictos.

Vera Carrera, J. M, \& Valdés-Espinosa, E. (2021). Pertinencia de la formación a los capacitadores electorales en materia de los métodos de solución de conflictos para 
generar de forma creativa soluciones a los conflictos electorales. Revista Eletronica de Direito Processual, pp. 444 - 464. Recuperado en:https://www.e-publicacoes. uerj.br/index.php/redp/article/view/56703/36327

\section{Eni Valdes-Espinosa}

Doctora en Métodos Alternos de Solución de Conflictos por la Universidad Autónoma de Nuevo León; Maestría en Educación Southern New Hamshire University, Maestría en Clínica Psicoanalítica Universidad Autónoma de Nuevo León, Psicoanalista, correo de contacto eni_valdes@hotmail.com https://orcid. org/0000-0003-1483-5386 
